Check for updates

Cite this: RSC Adv., 2018, 8, 13154

\title{
Thermoelectric properties of polycrystalline palladium sulfide
}

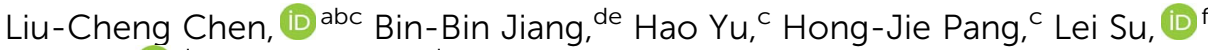 \\ Xun Shi, (DD ${ }^{d}$ Li-Dong Chen ${ }^{d}$ and Xiao-Jia Chen ${ }^{\star c}$
}

Received 23rd February 2018

Accepted 25th March 2018

DOI: 10.1039/c8ra01613e

rsc.li/rsc-advances

\begin{abstract}
Measurement of the electrical, thermal, and structural properties of palladium sulfide (PdS) has been conducted in order to investigate its thermoelectric performance. A tetragonal structure with the space group $P 4_{2} / \mathrm{m}$ for PdS was determined from $\mathrm{X}$-ray diffraction measurement. The obtained power factor of $27 \mu \mathrm{W} \mathrm{cm} \mathrm{cm}^{-1} \mathrm{~K}^{-2}$ at $800 \mathrm{~K}$ is the largest value obtained for the transition metal sulfides studied so far. The maximum value of the dimensionless figure of merit is 0.33 at $800 \mathrm{~K}$. These results indicate that binary bulk PdS has promising potential for good thermoelectric performance.
\end{abstract}

\section{Introduction}

Thermoelectric materials, which can directly convert heat to electrical power, have been of interest for many years because of their potential applications and environmentally friendly properties. The efficiency of thermoelectric materials is determined using the dimensionless figure of merit $(z T)$, defined as $z T=\frac{S^{2} \sigma}{\kappa} T$, where $S$ is the Seebeck coefficient, $\sigma$ is the electrical conductivity, $T$ is the absolute temperature and $\kappa$ is the thermal conductivity. For thermoelectric devices, a conversion efficiency of $15 \%(z T \geq 1)$ is needed and this can be achieved by obtaining large $S$, high $\sigma$, and low $\kappa$ values. ${ }^{1,2}$ Many strategies for enhancing $z T$ have been proven to be effective, including the use of phonon-liquid electron-crystal materials, nanostructure engineering and band structure engineering. ${ }^{3-5}$ Among these strategies, the reduction of thermal conductivity by microstructure modification is the most promising strategy. Therefore, searching for thermoelectric materials with intrinsic large power factors, PFs $\left(\mathrm{PF}=S^{2} \sigma\right)$, and then modifying them through nanostructuring while maintaining the original electrical properties will be a good method for obtaining superior performance in thermoelectric materials.

Recently, transition metal sulfides have attracted much attention because sulfide is cheaper and more earth abundant

${ }^{a}$ Key Laboratory of Materials Physics, Institute of Solid State Physics, Chinese Academy of Sciences, Hefei 230000, China

${ }^{b}$ University of Science and Technology of China, Hefei 230026, China

${ }^{c}$ Center for High Pressure Science and Technology Advanced Research, Shanghai 201203, China.E-mail: xjchen@hpstar.ac.cn

${ }^{d}$ State Key Laboratory of High Performance Ceramics and Superfine Microstructure, Shanghai Institute of Ceramics, Chinese Academy of Science, Shanghai 200050, China ${ }^{e}$ University of Chinese Academy of Sciences, Beijing 100049, China

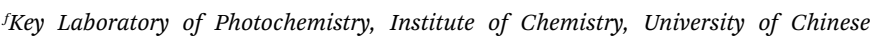
Academy of Sciences, Chinese Academy of Sciences, Beijing 100190, China than telluride or selenium. ${ }^{6,7}$ Many transition metal sulfides have shown good thermoelectric performance, mainly because of their relatively low thermal conductivities. For instance, the highest $z T$ for $\mathrm{PbS}$ has been improved from 0.4 to $\sim 1$ by obtaining a low thermal conductivity through microstructure modification..$^{8-10}$ The reduction of thermal conductivity led to a high $z T$ of 0.6 at $873 \mathrm{~K}$ for SnS through a doping method. ${ }^{11}$ Copper sulfide is an important thermoelectric material with high $z T \mathrm{~s}(z T \mathrm{~s}=1.4-1.7$ at $1000 \mathrm{~K})$. Ultra-low lattice thermal conductivities caused by liquid-like copper ions were proposed to account for such high $z T \mathrm{~s}$ in $\mathrm{Cu}_{x} \mathrm{~S} .{ }^{12}$ However, the PFs of those thermoelectric sulfides are not high enough to provide ideal $z T$ s. For example, the best performing material, $\mathrm{Cu}_{1.97} \mathrm{~S}$, only has a PF of around $8 \mu \mathrm{W} \mathrm{cm}{ }^{-1} \mathrm{~K}^{-2}$ at $1000 \mathrm{~K}$. Thus, an interesting approach for obtaining a high $z T$ value is to search for a thermoelectric sulfide with an intrinsically large PF. Palladium sulfide (PdS), which belongs to the transition metal sulfide group, has potential applications in semiconducting, photoelectrochemical and photovoltaic fields because of its ideal band gap of $1.6 \mathrm{eV}^{13-15}$ Furthermore, it also has several potential device applications in catalysis and acid resistant and high temperature electrodes. ${ }^{16-18}$ In a study of thermoelectric properties, ${ }^{19}$ the Seebeck coefficient of PdS thin film was reported to have a large value (about $280 \mu \mathrm{V} \mathrm{K} \mathrm{K}^{-1}$ ) at room temperature. Therefore in this study, it is highly desirable to investigate the bulk thermoelectric properties of PdS, with the purpose of exploring the viability of the sample as a potentially useful thermoelectric material. In addition, it is interesting to gain some insight into the physical mechanisms involved in enhancing the $z T \mathrm{~s}$ of thermoelectric materials.

In this work, polycrystalline PdS was successfully fabricated using a melt quenching and spark plasma sintering technique. Its thermoelectric properties were investigated using measurement of the electrical conductivity, Seebeck coefficient and thermal conductivity. We found that the power factor was very 
large ( $\mathrm{PF}=27 \mu \mathrm{W} \mathrm{cm}{ }^{-1} \mathrm{~K}^{-2}$ ), due to the high values of $\sigma$ and $S$. The highest $z T$ value achieved in this paper was 0.33 at $800 \mathrm{~K}$. Our results highlight that polycrystalline PdS is a promising potential thermoelectric material after overcoming the high $\kappa$ value with microstructure modification.

\section{Experimental details}

High purity raw elements, Pd (powder, 99.99\%, Alfa Aesar) and S (powder, 99.999\%, Alfa Aesar), were weighed out in stoichiometric proportions and then mixed well in an agate mortar. The mixture was pressed into pellets and sealed in quartz tubes under vacuum. Then, the tubes were heated at a rate of $1{ }^{\circ} \mathrm{C} \min ^{-1}$ to $1373 \mathrm{~K}$. They remained at this temperature for 12 hours before quenching in cold water. Next, the quenched tubes were annealed at $873 \mathrm{~K}$ for 7 days. Finally, the products were ground into fine powders and sintered by Spark Plasma Sintering (Sumitomo SPS-2040) at $923 \mathrm{~K}$ under a pressure of $65 \mathrm{MPa}$ for $5 \mathrm{~min}$. High-density samples $(>99 \%$ of the theoretical density) were obtained.

The powders were characterized using X-ray diffraction (XRD) (Rigaku, Rint 2000) under $\mathrm{Cu} \mathrm{K} \alpha$ radiation $(\lambda=1.5405 \AA)$ at room temperature. Measurements were obtained between $20^{\circ}$ and $70^{\circ}$ with a scan width of $0.02^{\circ}$ and a rate of $2^{\circ} \mathrm{min}^{-1}$. The Seebeck coefficient and electrical and thermal conductivities were simultaneously measured between $3 \mathrm{~K}$ and $300 \mathrm{~K}$ in a thermal transport option (TTO) setup using a Physical Properties Measurement System (PPMS) by Quantum Design. The measurements were carried out in the residual vacuum of a He atmosphere, under a pressure of $10^{-5}$ Torr. The typical size of PdS used in the PPMS was $4.3 \times 2.0 \times 0.9 \mathrm{~mm}^{3}$, with four $\mathrm{Cu}$ wires attached with $\mathrm{Ag}$ paste. The Hall coefficient, $R_{\mathrm{H}}$, was also measured using a conventional four-probe technique by the PPMS, with a temperature range of $7 \mathrm{~K}$ to $300 \mathrm{~K}$. The heat capacity, $C_{\mathrm{p}}$, in a temperature range of 1.8 to $300 \mathrm{~K}$ was additionally measured by the PPMS in order to analyze the thermal conductivity data. The high temperature electrical conductivity and the Seebeck coefficient were measured using an Ulvac ZEM3 from 300 to $800 \mathrm{~K}$ under a helium atmosphere. The high temperature thermal conductivity was calculated from $\kappa=D C_{\mathrm{p}} \rho$, where the thermal diffusivity $(D)$ was obtained using a laser flash method (Netzsch, LFA 457) under an argon atmosphere. The specific heat $\left(C_{\mathrm{p}}\right)$ was calculated using the Dulong-Petit law, and the density $(\rho)$ was measured using the Archimedes method.

\section{Results and discussion}

\subsection{Electrical transport properties}

The XRD pattern and crystal structure of bulk PdS are shown in Fig. 1. The main diffraction peaks indicate a tetragonal structure (JCPDS no. 25-1234) with the space group $P 4_{2} / m$ (84) and no other phases were obviously detected from the XRD pattern. The lattice parameters are $a=b=6.441 \AA$ and $c=6.619 \AA$.

The temperature dependences of the $\sigma, S$, and PF of polycrystalline PdS are shown in Fig. 2. It can be seen that the values for $\sigma, S$ and PF measured in different studies are consistent with each other. The small deviations of the data around room

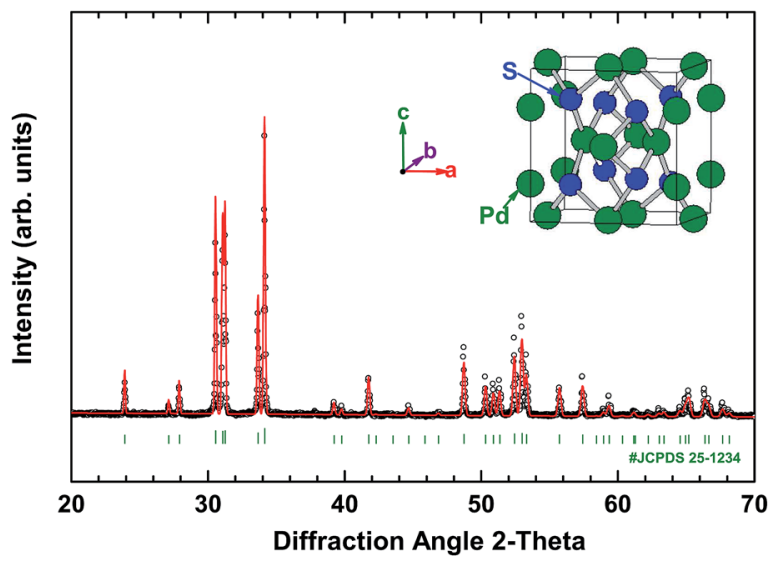

Fig. 1 Powder XRD pattern of the PdS sample at room temperature. The inset illustrates the crystal structure of PdS.

temperature could be caused by the different errors of the two systems. In Fig. 2(a), the behavior of $\sigma$ with changing temperature is complex. At high temperatures, $\sigma$ decreases sharply with decreasing temperature until around $500 \mathrm{~K}$, which is probably caused by the thermal activation induced bipolar effect in semiconductors. However, in the temperature range of 200-500 K, $\sigma$ behaves like a constant. It does not show an obvious increase with decreasing temperature. This behavior is consistent with that of a metal, which was not observed previously. ${ }^{14}$ Below $200 \mathrm{~K}$, there is a significant decrease in the temperature dependence of $\sigma$, decreasing sharply as the sample is cooled. This reflects the nature of a typical semiconductor. This behavior at low temperatures can be seen more obviously in the inset [Fig. 2(a)]. Therefore, these contrasting behaviors imply that bulk PdS may have semiconductor-metal and metal-semiconductor transitions around $200 \mathrm{~K}$ and $500 \mathrm{~K}$, respectively.

In Fig. 2(b), the temperature dependences of $S$ and PF are shown. The value of $S$ is negative, indicating that the majority of the charge carriers are electrons. With increasing temperature, the absolute value of $S$ increases to a very large value at approximately $400 \mu \mathrm{V} \mathrm{K}^{-1}$, without doping at around $350 \mathrm{~K}$, and then reaches a plateau until about $600 \mathrm{~K}$. Unfortunately, there is an obvious decrease above $600 \mathrm{~K}$, which may be caused by the thermal excitation of the carriers. This behavior observed for $\sigma$ and $S$ at high temperatures has been observed in many other intrinsic semiconductor systems and may be ameliorated by doping. ${ }^{20}$ Notably, the PF value tends to increase over the entire temperature range, except the intermediate region. The maximum $P F$ value is about $27 \mu \mathrm{W} \mathrm{cm}{ }^{-1} \mathrm{~K}^{-2}$ at $800 \mathrm{~K}$ (the highest recorded temperature in this study). The $\mathrm{PF}$ value reported here is very large compared with values obtained for other thermoelectric sulfides. For example, it is about three times larger than the value of optimal $\mathrm{Cu}_{1.97} \mathrm{~S} .{ }^{12}$ From these electrical properties, it is obvious that PdS is a potentially useful thermoelectric material.

The Hall effect was measured to give insight into the electrical transport properties of PdS. The temperature dependences of the carrier concentration $\left(n_{\mathrm{H}}\right)$ and Hall mobility $\left(\mu_{\mathrm{H}}\right)$, evaluated from low temperature Hall measurements, are shown in Fig. 3. The Hall coefficient $\left(R_{\mathrm{H}}\right)$ is negative over the entire 

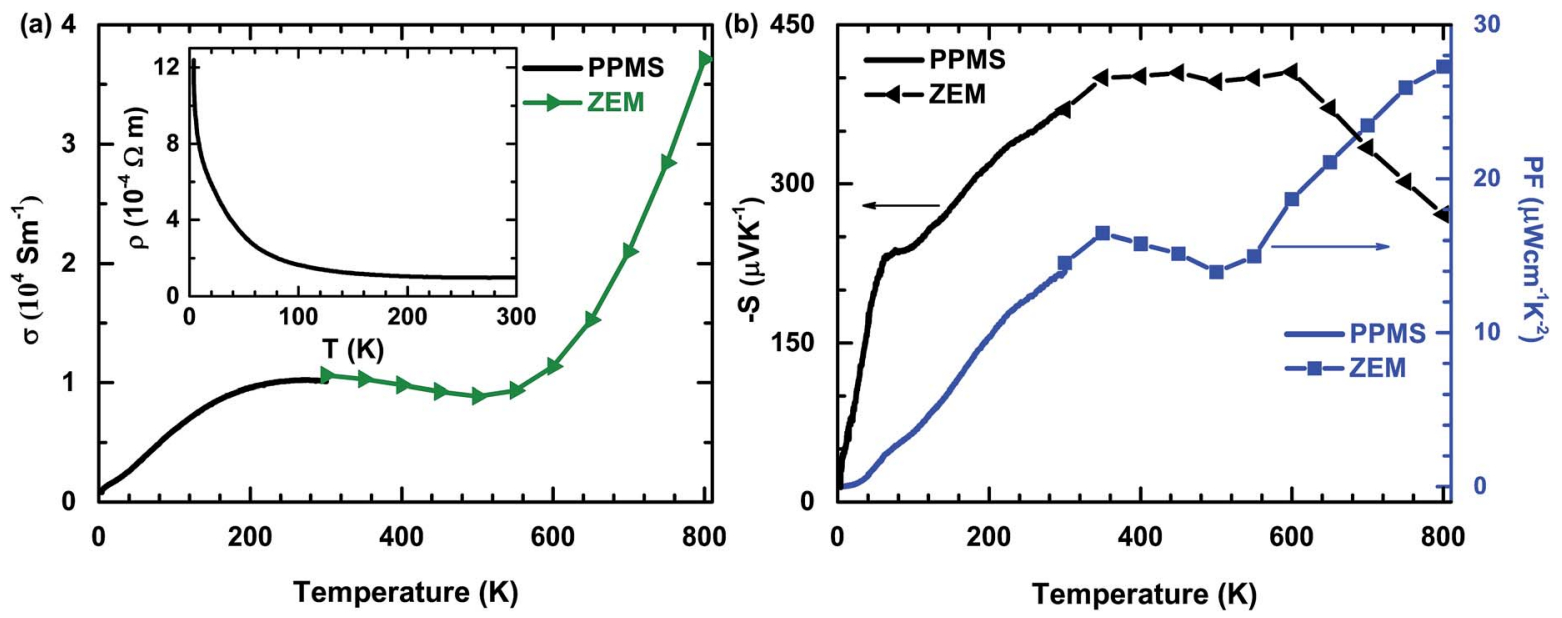

Fig. 2 Temperature dependence of the electrical transport properties of bulk PdS; (a) temperature dependence of the electrical conductivity ( $\sigma$ ) from 3 to $800 \mathrm{~K}$. The inset shows $\rho$ versus $T$ below room temperature. (b) Temperature dependence of the Seebeck coefficient (S) and the calculated power factor (PF $=S^{2} \sigma$ ) from 3 to $800 \mathrm{~K}$.

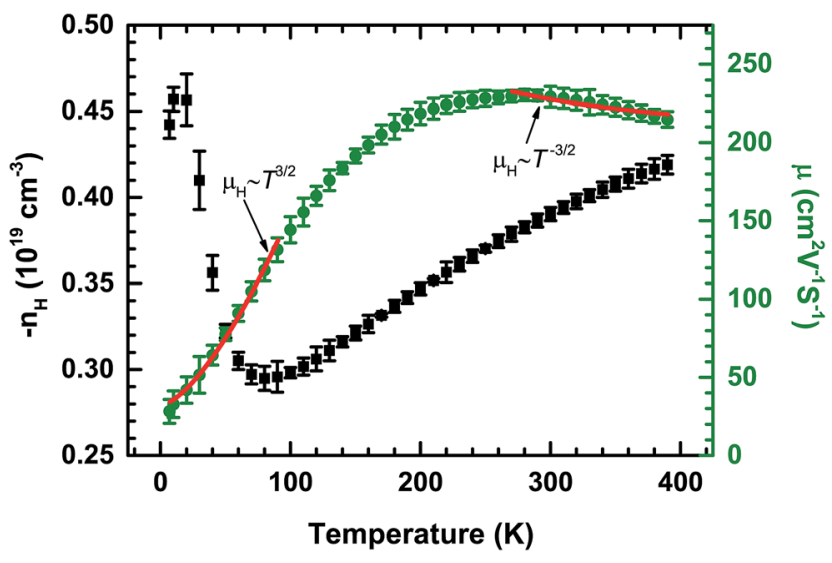

Fig. 3 Temperature dependences of the Hall carrier concentration $\left(n_{H}\right)$ for PdS from $6 \mathrm{~K}$ to $300 \mathrm{~K}$ (black line) and the Hall mobility $\left(\mu_{H}\right)$ (olive line) in the same temperature range. The red solid lines are fitted curves, $\mu_{\mathrm{H}} \sim T^{3 / 2}$ for low temperatures and $\mu_{\mathrm{H}} \sim T^{-3 / 2}$ for high temperatures.

temperature range, indicating that the majority of the charge carriers are electrons, which is consistent with the negative Seebeck coefficient. The temperature dependent $\mu_{\mathrm{H}}$ gently increases until around $200 \mathrm{~K}$ and the maximum value is $230 \mathrm{~cm}^{2}$ $\mathrm{V}^{-1} \mathrm{~s}^{-1}$. This relatively high value of $\mu_{\mathrm{H}}$ could be caused by the covalent bond characteristics of the tetragonal structure. Then, this value remains almost unchanged up to $300 \mathrm{~K}$. This behavior is consistent with the temperature dependence of $\sigma$ below 300 $\mathrm{K}$, as shown in Fig. 2(a). At low temperatures, $\mu_{\mathrm{H}}$ fits the curve
$\mu_{\mathrm{H}} \sim T^{3 / 2}$ well, which indicates that ionized impurity scattering is the dominating carrier scattering process. However, at high temperatures, a $T^{-3 / 2}$ dependence is observed in PdS, suggesting that the major carrier scattering process has changed to acoustic phonon scattering. The temperature dependence of $n_{\mathrm{H}}$ is very complex and incredible, especially at low temperatures, which is most likely caused by some magnetic transitions. However, the carrier concentration of PdS changes in the same order, which indicates that the carrier concentration for PdS has a weak temperature dependence below $300 \mathrm{~K}$, which has also been observed in lead chalcogenides. ${ }^{21}$

\subsection{Heat transport properties}

The temperature dependences of the thermal diffusivity and specific heat capacity used for the $\kappa$ calculation are shown in Table 1 . The obtained values for $\kappa$ with changing temperatures are shown in Fig. 4. It can be seen that $\kappa$ increases sharply with increasing temperature and evolves through a maximum (about $130 \mathrm{~W} \mathrm{~m}^{-1} \mathrm{~K}^{-1}$ ) at $38 \mathrm{~K}$ before finally decreasing roughly in a $T^{-1}$ relation. This phenomenon indicates that bulk PdS is a normal crystal compound. Generally, $\kappa$ consists of the electronic part, $\kappa_{\text {ele }}$, and the lattice part, $\kappa_{\text {lat }}$. The electronic part $\kappa_{\text {ele }}$ is proportional to electrical conductivity $\sigma$ through the Wiedemann-Franz relation: $:^{22} \kappa_{\text {ele }}=L \sigma T$, where $L$ is the Lorenz number $\left(L=2.44 \times 10^{-8} \mathrm{~W} \Omega \mathrm{K}^{-2}\right.$ in theory for semiconductors). Here, $\kappa_{\text {ele }}$ can be ignored below room temperature, as shown in the inset of Fig. 4 . However, the contribution of $\kappa_{\text {ele }}$ to the total $\kappa$ increases with increasing temperature and reaches

Table 1 Summary of the values for thermal diffusivity, $D$, heat capacity, $C_{p}$, and density, $d$, which were used to calculate the thermal conductivity, $\kappa$, of PdS

\begin{tabular}{llllllllllll}
\hline$T(\mathrm{~K})$ & 300 & 350 & 400 & 450 & 500 & 550 & 600 & 650 & 700 & 750 & 800 \\
$D\left(\mathrm{~cm}^{2} \mathrm{~s}^{-1}\right)$ & 10.137 & 8.328 & 7.046 & 6.015 & 5.216 & 4.602 & 4.083 & 3.67 & 3.305 & 3.035 & 2.817 \\
$C_{\mathrm{p}}\left(\mathrm{J} \mathrm{g}^{-1} \mathrm{~K}^{-1}\right)$ & 0.36 & 0.36 & 0.36 & 0.36 & 0.36 & 0.36 & 0.36 & 0.36 & 0.36 & 0.36 & 0.36
\end{tabular}




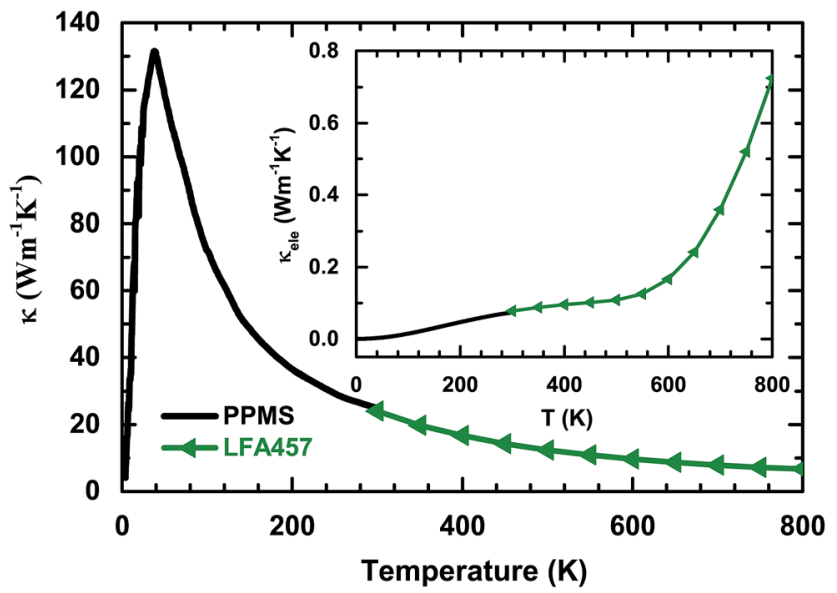

Fig. 4 Temperature dependence of the total thermal conductivity $(\kappa)$ of bulk PdS. The inset illustrates the electric thermal conductivity $\left(\kappa_{\text {ele }}\right)$ vs. $T$ plot in the temperature range between 3 and $800 \mathrm{~K}$.

about $12 \%$ at $800 \mathrm{~K}$. The high $\kappa$ of PdS probably comes from the light atomic mass of sulfur and the strong chemical bonds in the crystal. In order to elucidate the reasons for the high thermal conductivity of PdS, the heat capacity, $C_{\mathrm{p}}$, was measured at low temperatures. The results are shown in Fig. 5. The measured $C_{\mathrm{p}}$ value at $300 \mathrm{~K}$ is $0.35 \mathrm{~J} \mathrm{~g}^{-1} \mathrm{~K}^{-1}$, which is close to the theoretical value $\left(0.36 \mathrm{~J} \mathrm{~g}^{-1} \mathrm{~K}^{-1}\right)$. The inset of Fig. 5 displays the heat capacity $\left(C_{\mathrm{p}}\right) v s$. $T$. The solid red line is a fitted curve based on the Debye model by the relation: ${ }^{23} C_{\mathrm{p}}=\varphi T+\beta T^{3}$. The total $C_{\mathrm{p}}$ includes the carrier contribution, $\varphi T$, and the phonon contribution, $\beta T^{3}$. The fitted parameters are $0.34 \mathrm{~mJ}$ $\mathrm{mol}^{-1} \mathrm{~K}^{-2}$ for $\varphi$ and $0.13 \mathrm{~mJ} \mathrm{~mol}^{-1} \mathrm{~K}^{-2}$ for $\beta$. The small value for $\varphi$ indicates that the electronic density of states near the Fermi level is quite weak at low temperatures when compared with other thermoelectric materials $\left(\right.$ e.g. $\mathrm{YbFe}_{4} \mathrm{Sb}_{12}, \varphi=141.2$ $\left.\mathrm{mJ} \mathrm{mol}{ }^{-1} \mathrm{~K}^{-2}\right){ }^{24}$ This finding is consistent with the low $\sigma$ observed at low temperatures, as shown in Fig. 2(a).

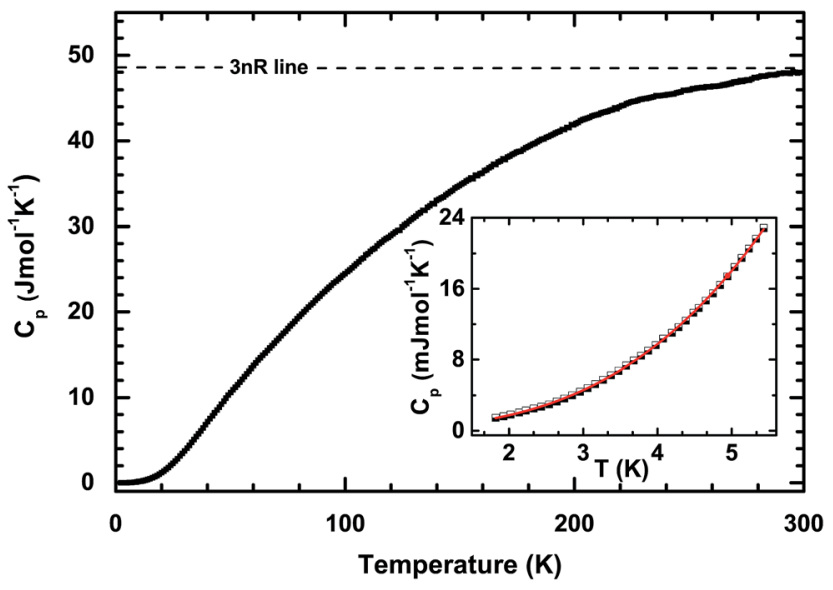

Fig. 5 Temperature dependence of the heat capacity $\left(C_{p}\right)$ for bulk PdS from $1.8 \mathrm{~K}$ to $300 \mathrm{~K}$. The dashed line is the $3 \mathrm{nR}$ line calculated using the Dulong-Petit model. The inset shows the heat capacity below $6 \mathrm{~K}$. The solid red line is the fitted curve, calculated using $C_{p}=\varphi T+\beta T^{3}$.

\subsection{The dimensionless figure of merit}

Based on the measured temperature-dependent values for $S, \sigma$ and $\kappa$, the dimensionless figure of merit $(z T)$, which directly determines the energy conversion efficiency of a thermoelectric material, has been calculated. The results are shown in Fig. 6 . The calculated value of $z T$ has a positive temperature dependence over the entire measurement range, which is different from other thermoelectric materials which have maximum peaks at suitable temperatures. This phenomenon indicates that the thermoelectric properties of bulk PdS will be more efficient at higher temperatures. The value of $z T$ reaches 0.33 at a temperature of $800 \mathrm{~K}$ (the highest recorded temperature in this study). This value is considerably high compared to that of many undoped thermoelectric sulfides at similar temperatures, such as $\mathrm{Bi}_{2} \mathrm{~S}_{3}(z T \sim 0.2$ at $823 \mathrm{~K}) .{ }^{25}$ However, the thermoelectric conversion efficiency is still low compared to that of high performance thermoelectric materials, despite the obvious uptrend at higher temperatures. Thus, it is extremely urgent to improve the thermoelectric conversion efficiency of bulk PdS.

Based on our results, the intrinsic large PF is the most important feature for bulk PdS. Since only nominally undoped samples were studied in this initial report, it's not hard to infer that further improvement of the PF through optimized doping should be a good method. This could change the density of states at the Fermi level depending on the relation: ${ }^{26}$ $S=\frac{\pi^{2}}{3} \frac{k_{\mathrm{B}}^{2} T}{q}\left[\frac{\mathrm{d} \ln \sigma(E)}{\mathrm{d} E}\right]_{E=E_{\mathrm{F}}}\left(q\right.$ is the carrier charge and $E_{\mathrm{F}}$ is the Fermi energy). Another way to improve the power factor of PdS is through band structure engineering, as many studies have explored in recent years. For example, the $z T$ of PbTe reached 1.5 at $773 \mathrm{~K}$ by distortion of the electronic density of states, ${ }^{26}$ and a higher $z T$ value of $\sim 1.8$ was observed in $\mathrm{PbTe}_{1-x} \mathrm{Se}_{x}$ alloys by producing the convergence of many valleys at desired temperatures. Furthermore, a successful approach of rationally tuning crystal structures in non-cubic materials has been proposed, which has enhanced the $z T$ values of a few carefully selected chalcopyrites. ${ }^{27}$ In addition, it can be expected that

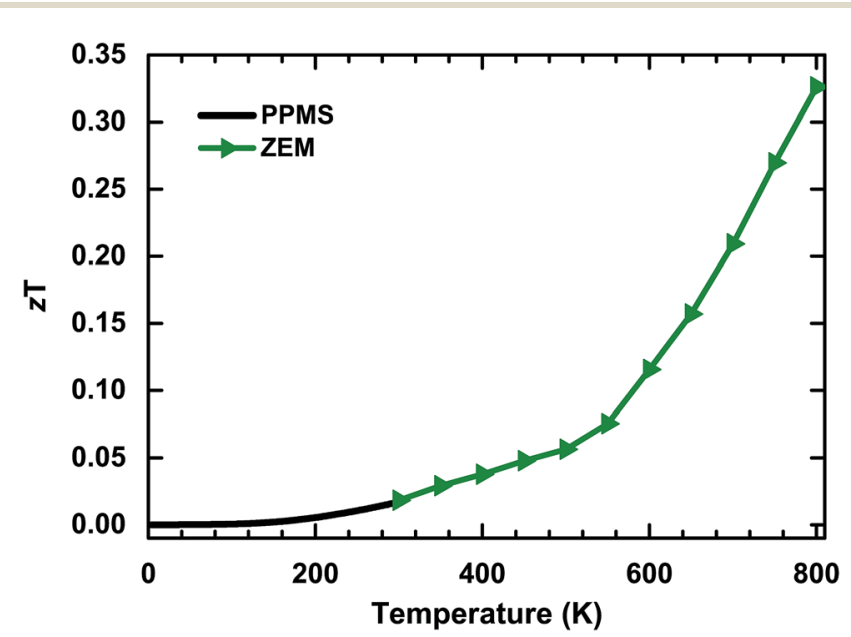

Fig. 6 Temperature dependence of the dimensionless figure of merit $(z T)$ from $2 \mathrm{~K}$ to $800 \mathrm{~K}$. The maximum value of $z T$ is 0.33 at $800 \mathrm{~K}$. 
alloying between $\mathrm{Pd}$ and $\mathrm{S}$ will lead to a reduction in $\kappa_{\text {lat }}$ due to the alloy scattering effect.

At the same time, the $\kappa$ of bulk PdS is too large compared to that of other thermoelectric materials with low thermal conductivity (Fig. 4). Therefore, the reduction of $\kappa$ appears to be very important and useful for the purpose of improving $z T$. Alloying and nanostructuring are both optional routes to increase phonon scattering for the purpose of reducing $\kappa$ effectively. ${ }^{2}$ Many thermoelectric materials have been improved by these methods, such as the analogous binary $\mathrm{PbS}$, where the $z T$ value of the nanostructured $\mathrm{PbS}$ is about twice as high as previously reported $(z T \sim$ $0.4){ }^{9}$ SiGe is a well-known alloy for high temperature thermoelectric applications. Recently, by controlling their nanoscale structures, the $z T$ values of both p- and n-type SiGe have been enhanced. $^{28,29}$ More importantly, nanostructuring has been proven to be an efficient method for lowering thermal conductivities and will not change the electrical transport properties too much. ${ }^{4}$ Therefore, controlling the nanostructure of binary PdS will be a useful way to improve the $z T$ value.

\section{Conclusions}

In summary, measurement of the electrical and thermal transport properties of binary PdS has been carried out in the temperature range of $2-800 \mathrm{~K}$. The large value of the power factor $\left(27 \mu \mathrm{W} \mathrm{cm}{ }^{-1} \mathrm{~K}^{-2}\right)$ and the maximum value of $z T$ of 0.33 at about $800 \mathrm{~K}$ indicate the great potential thermoelectric performance of PdS as an undoped thermoelectric material. This study also predicts that $\kappa_{\text {lat }}$ could be reduced through increased phonon scattering, which can be realized by nanostructuring, alloying or doping. These results suggest that binary bulk PdS has suitable properties to be a potential base thermoelectric material and many PdS based materials are expected to have good performance for thermoelectric applications.

\section{Conflicts of interest}

There are no conflicts to declare.

\section{Acknowledgements}

Lei Su acknowledges support from the Natural Science Foundation of China (No. 21273206). Xun Shi and Li-Dong Chen acknowledge support from the National Basic Research Program of China (973-program) under Project No. 2013CB632501, the Natural Science Foundation of China under No. 11234012 and the Shanghai Government (Grant No. 15JC1400301).

\section{Notes and references}

1 L. E. Bell, Science, 2008, 321, 1457-1461.

2 G. J. Snyder and E. S. Toberer, Nat. Mater., 2008, 7, 105-114. 3 B. C. Sales, D. Mandrus and R. K. Williams, Science, 1996, 272, 1325-1328.

4 K. Biswas, J. He, I. D. Blum, C. I. Wu, T. P. Hogan, D. N. Seidman, V. P. Dravid and M. G. Kanatzidis, Nature, 2012, 489, 414-418.
5 Y. Z. Pei, X. Shi, A. Lalonde, H. Wang, L. D. Chen and G. J. Snyder, Nature, 2011, 473, 66-69.

6 X. Lu, D. T. Morelli, Y. Xia, F. Zhou, V. Ozolins, H. Chi, X. Y. Zhou and C. Uher, Adv. Energy Mater., 2013, 3, 342-348.

7 C. Wan, Y. Wang, N. Wang, W. Norimatsu, M. Kusunoki and K. Koumoto, Adv. Mater., 2010, 11, 044306.

8 L. D. Zhao, S. H. Lo, J. He, H. Li, K. Biswas, J. Androulakis, C. I. Wu, T. P. Hogan, D. Y. Chung and V. P. Dravid, J. Am. Chem. Soc., 2011, 133, 20476-20487.

9 S. Johnsen, J. He, J. Androulakis, V. P. Dravid, I. Todorov, D. Y. Chung and M. G. Kanatzidis, J. Am. Chem. Soc., 2011, 133, 3460-3470.

10 L. D. Zhao, J. He, S. Hao, C. I. Wu, T. P. Hogan, C. Wolverton, V. P. Dravid and M. G. Kanatzidis, J. Am. Chem. Soc., 2012, 134, 16327-16336.

11 Q. Tan, L. D. Zhao, J. F. Li, C. F. Wu, T. R. Wei, Z. B. Xing and M. G. Kanatzidis, J. Mater. Chem. A, 2014, 2, 17302-17306.

12 Y. He, T. Day, T. Zhang, H. Liu, X. Shi, L. D. Chen and G. J. Snyder, Adv. Mater., 2014, 26, 3974-3978.

13 J. C. W. Folmer, J. A. Turner and B. A. Parkinson, J. Solid State Chem., 1987, 68, 28-37.

14 I. J. Ferrer, P. Díaz-Chao, A. Pascual and C. Sánchez, Thin Solid Films, 2007, 515, 5783-5786.

15 M. Barawi, I. J. Ferrer, J. R. Ares and C. Sánchez, ACS Appl. Mater. Interfaces, 2014, 6, 20544-20549.

16 J. J. Bladon, J. Electrochem. Soc., 1996, 143, 1206-1213.

17 A. Zubkov, T. Fujino, N. Sato and K. Yamada, J. Chem. Thermodyn., 1998, 30, 571-581.

18 C. H. Yang, Y. Y. Wang, C. C. Wan and C. J. Chen, J. Electrochem. Soc., 1996, 143, 3521-3525.

19 A. Pascual, J. R. Ares, I. J. Ferrer, and C. R. Sanchez, in International Conference on - ICT, 2003, pp. 376-379.

20 Y. Z. Pei, J. Lensch Falk, E. S. Toberer, D. L. Medlin and G. J. Snyder, Adv. Funct. Mater., 2011, 21, 241-249.

21 W. W. Scanlon, Solid State Phys., 1959, 9, 83.

22 G. S. Kumar, G. Prasad and R. O. Pohl, J. Mater. Sci., 1993, 28, 4261-4272.

23 K. Gofryk, D. Kaczorowski, T. Plackowski, J. Mucha, A. Leithejasper, W. Schnelle and Y. Grin, Phys. Rev. B: Condens. Matter Mater. Phys., 2007, 75, 1-10.

24 N. R. Dilley, E. D. Bauer, M. B. Maple, S. Dordevic, D. N. Basov, F. Freibert, T. W. Darling, A. Migliori, B. C. Chakoumakos and B. C. Sales, Phys. Rev. B: Condens. Matter Mater. Phys., 2000, 61, 4608-4614.

25 Z. H. Ge, B. P. Zhang, Z. X. Yu and J. F. Li, J. Mater. Res., 2011, 26, 2711-2718.

26 J. P. Heremans, V. Jovovic, E. S. Toberer, A. Saramat, K. Kurosaki, A. Charoenphakdee, S. Yamanaka and G. J. Snyder, Science, 2008, 321, 554-557.

27 J. Zhang, R. Liu, N. Cheng, Y. Zhang, J. Yang, C. Uher, X. Shi, L. D. Chen and W. Zhang, Adv. Mater., 2014, 26, 3848-3853. 28 X. W. Wang, H. Lee, Y. C. Lan, G. H. Zhu, G. Joshi, D. Z. Wang, J. Yang, A. J. Muto, M. Y. Tang and J. Klatsky, Appl. Phys. Lett., 2008, 93, 193121.

29 G. Joshi, H. Lee, Y. C. Lan, X. W. Wang, G. H. Zhu, D. Z. Wang, R. W. Gould, D. C. Cuff, M. Y. Tang and M. S. Dresselhaus, Nano Lett., 2008, 8, 4670-4674. 